\title{
Early-Onset Cerebral Amyloid Angiopathy, A Prion-Like Disease: Case Report and Literature Review
}

\author{
Hiria Limpo, $M D^{1 *}$, Alberto Andrés, $M D^{2}$, José Fortes, $M D^{3}$, María A García, MD² and Anna Lo Presti, $M D^{1}$ \\ ${ }^{1}$ Department of Neurosurgery, Fundación Jiménez Díaz University Hospital, Spain \\ ${ }^{2}$ Department of Neurology, Fundación Jiménez Díaz University Hospital, Spain \\ ${ }^{3}$ Department of Pathology, Fundación Jiménez Díaz University Hospital, Spain

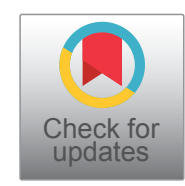

\begin{abstract}
Background: Cerebral amyloid angiopathy is an extremely rare pathology in young patients. In this group of patients, humanto-human amyloid- $\beta$ transmission has been hypothesized, following neurosurgical procedures carried out years before.

Case Description: We report the case of a 38-year-old woman who suffered repeated intracerebral hemorrhages caused by cerebral amyloid angiopathy three decades after an astrocytoma resection with cadaveric dural graft used for closing. Neuroimaging showed extensive left parenchymal hematoma, several microbleeds and frontal and occipital superficial siderosis. Pathology showed blood vessels with deposits stained with Congo Red, suggestive of cerebral amyloid angiopathy. Genetic tests did not reveal any mutation related to hereditary forms of $\beta$-amyloid pathology.

Conclusion: This case report highlights the importance of considering cerebral amyloid angiopathy as part of differential diagnosis of lobar intracerebral hemorrhage in young adults, especially in those with a past medical history of neurosurgery and provide new evidence to support that amyloid- $\beta$ might be transmitted as a prion disease.
\end{abstract}

\section{Keywords}

Cerebral amyloid angiopathy, Dural graft, Neurosurgical procedures, Prion, latrogenic Creutzfeldt- Jakob disease, Amyloid- $\beta$

\section{Introduction}

Cerebral amyloid angiopathy (CAA) is a progressive form of microvascular amyloidosis frequently responsible for recurrent lobar hemorrhages in older patients. Few cases have been reported in patients aged $<55$-years. There is evidence suggesting that in young patients, amyloid- $\beta(A \beta)$, the hallmark of CAA, might be transmitted by a similar mechanism to acquired prion disease[1-3]. Initially described in a patient diagnosed with iatrogenic Creutzfelt-Jakob disease (iCJD) after dural grafting [4], $A \beta$ transmission has been also reported in patients without iCJD after neurosurgery [1,2,4-7].

\section{Case Report}

A 38-year-old woman presented with sudden aphasia, right hemianopsia and hemiparesis. Her past medical history included a resection of an astrocytoma at the age of 4 , using cadaveric dural graft for closure. Family history of Alzheimer's disease (AD) or CAA could not have been documented, as she was adopted at birth. Computed Tomography (CT) revealed an acute spontaneous left temporo-parieto-occipital hematoma managed conservatively. Electroencephalography demonstrated left fronto-temporal slowing in relation to underlying lesion. Carotid duplex, brain MRI-angiography, transthoracic echocardiography, 24-hour electrocardiogram and coagulation study did not reveal relevant findings. A left inferior quadrantanopsy and mild dysphasia at discharge persisted.

Six months later, the patient presented with sudden blindness and headache that rapidly progressed into a coma. CT brain showed a right temporo-occipital intraparenchymatous hematoma with midline deviation that required evacuation and decompressive craniectomy. T1 MRI sequences revealed lobar right temporo-occipital hemorrhage. (Figure 1A). Superficial siderosis and several microbleeds were observed in susceptibility weighted imaging (SWI) sequences (Figure 1B). Pathology of the evacuated haematoma (Figure 1C) showed

*Corresponding authors: Hiria Limpo, MD, Department of Neurosurgery, Fundación Jiménez Díaz University Hospital, Av. Reyes Católicos, 2. 28008, Spain, Tel: +34609276774

Accepted: December 29, 2021

Published online: December 31, 2021

Citation: Limpo H, Andrés A, Fortes J, et al. (2021) Early-Onset Cerebral Amyloid Angiopathy, A Prion-Like Disease: Case Report and Literature Review. J Neurosurg Res Rev 4(1):72-75 

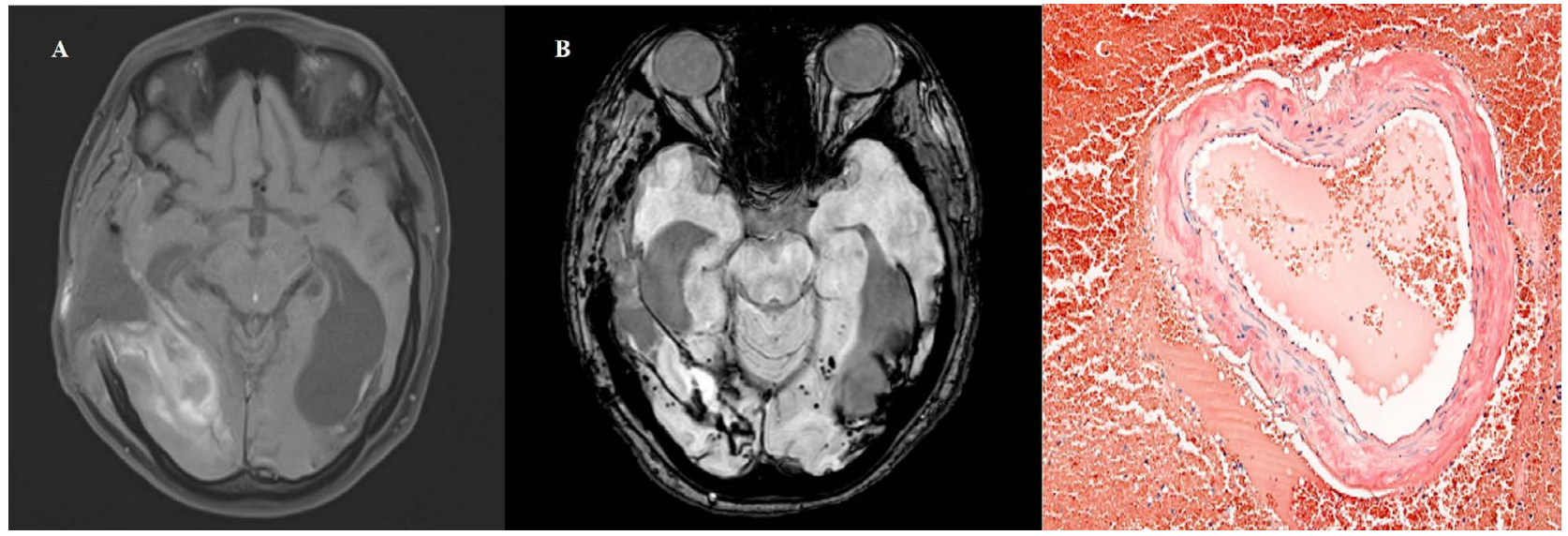

Figure 1: (A) T1 MRI sequence showing recurrent lobar hemorrhage with radiological stability of previous contralateral lesions. (B) SWI sequence demonstrating superficial siderosis and microbleeds. (C) Deposits of Congo Red in a cerebral blood vessel arterial wall.

Table 1: Patients who underwent a neurosurgical procedure prior to develop CAA and/or iCJD.

\begin{tabular}{|c|c|c|c|c|c|c|c|c|}
\hline Author & Age & Sex & Presentation & $\begin{array}{l}\text { Documented } \\
\text { Dural Graft } \\
\text { (cadaveric or } \\
\text { synthetic) }\end{array}$ & $\begin{array}{l}\text { Neurosurgical } \\
\text { Procedure }\end{array}$ & $\begin{array}{l}\text { Interval of Years } \\
\text { from the Surgery } \\
\text { to the Illness }\end{array}$ & $\begin{array}{l}\text { Genetics: } \\
\text { APP, } \\
\text { PSEN1, } \\
\text { PSEN2 }\end{array}$ & $\begin{array}{l}\text { Genetics: } \\
\text { APOEE }\end{array}$ \\
\hline Ehling [9] & 38 & M & CAA & No & TBI & $>20$ & NM & $\varepsilon 3 / \varepsilon 3$ \\
\hline Purruker [11] & 37 & M & CAA & No & TBI & $>20$ & NM & NA \\
\hline Purruker [11] & 42 & $M$ & CAA & No & TBI & 40 & NM & NA \\
\hline Frontzek & 28 & M & $i C J D+C A A$ & Yes & TBI & 21 & NM & NA \\
\hline Frontzek [8] & 33 & $\mathrm{~F}$ & $i C J D+C A A$ & Yes & TBI & 25 & NM & NA \\
\hline Frontzek [8] & 47 & M & $i C J D+C A A$ & Yes & TBI & 21 & NM & NA \\
\hline Frontzek [8] & 51 & $M$ & iCJD & Yes & Not specified & 12 & NM & NA \\
\hline Frontzek [8] & 52 & $F$ & $i C J D+C A A$ & Yes & Pituitare adenoma & 22 & NM & NA \\
\hline Nakayama [7] & 32 & $M$ & CAA & No & $\mathrm{TBI}$ & 31 & NM & $\varepsilon 3 / \varepsilon 3$ \\
\hline Jaunmuktane [2] & 39 & $\mathrm{~F}$ & CAA & No & TBI & 36 & NM & $\varepsilon 3 / \varepsilon 4$ \\
\hline Jaunmuktane [8] & 31 & $M$ & CAA & No & Brain tumor & 30 & NM & $\varepsilon 2 / \varepsilon 3$ \\
\hline Jaunmuktane [8] & 36 & $\mathrm{~F}$ & CAA & No & $\begin{array}{l}\text { Craneofacial } \\
\text { malformations }\end{array}$ & 35 & NM & $\varepsilon 3 / \varepsilon 4$ \\
\hline Hervé [1] & 46 & $F$ & CAA & Yes & $\mathrm{TBI}$ & 44 & NM & $\varepsilon 2 / \varepsilon 3$ \\
\hline Cali [12] & 26 & M & iCJD & Yes & Not specified & 19 & NM & NA \\
\hline Cali [12] & 39 & $\mathrm{~F}$ & iCJD & Yes & Not specified & 6 & NM & NA \\
\hline Cali [12] & 32 & M & iCJD & Yes & Not specified & 16 & NM & NA \\
\hline Cali [12] & 25 & $M$ & iCJD & Yes & Not specified & 8 & NM & NA \\
\hline Cali [12] & 29 & $M$ & iCJD & Yes & Not specified & 25 & NM & NA \\
\hline Cali [12] & 42 & $M$ & iCJD & Yes & Not specified & 6 & NM & NA \\
\hline Cali [12] & 50 & $\mathrm{~F}$ & iCJD & Yes & Not specified & 11 & NM & NA \\
\hline Cali [12] & 23 & $\mathrm{~F}$ & iCJD & Yes & Not specified & 21 & NM & NA \\
\hline Cali [12] & 26 & $\mathrm{~F}$ & iCJD & Yes & Not specified & 12 & NM & NA \\
\hline Cali [12] & 42 & $M$ & iCJD & Yes & Not specified & 18 & NM & NA \\
\hline Banerjee [5] & 48 & $M$ & CAA & Yes & Brain tumor & 37 & NM & $\varepsilon 3 / \varepsilon 3$ \\
\hline Banerjee [5] & 39 & M & CAA & Yes & $\begin{array}{l}\text { Cavernous } \\
\text { hemangioma } \\
\text { embolization }\end{array}$ & 37 & NM & $\varepsilon 2 / \varepsilon 3$ \\
\hline Banerjee [5] & 34 & $F$ & CAA & Yes & $\mathrm{TBI}$ & 34 & NM & $\varepsilon 3 / \varepsilon 3$ \\
\hline Hamaguchi [6] & 30 & $M$ & CAA & No & TBI & 30 & NM & $\varepsilon 3 / \varepsilon 3$ \\
\hline Hamaguchi [6] & 30 & $M$ & CAA & No & TBI & 30 & NM & $\varepsilon 3 / \varepsilon 3$ \\
\hline Giaccone [10] & 29 & $M$ & CAA & No & TBI & 28 & NM & NA \\
\hline
\end{tabular}

$\mathrm{NA}=$ Not Available; $\mathrm{TB}=$ Trauma Brain Injury; $\mathrm{CAA}=$ Cerebral Amyloid Angiopathy $\mathrm{CJD}=$ Creuzfelt-Jakob Disease, $\mathrm{NM}=$ No Mutations 
blood vessels with deposits stained with Congo Red, suggestive of amyloid angiopathy. Sequencing for mutations in genes associated with hereditary forms of CCA and Alzheimer's disease (APP, CST3 and ITMB2) was negative. The bone flap was replaced and at six months follow up the patient remains with visual deficit and mild cognitive impairment.

\section{Literature Review}

The Ovid MEDLINE (1946 to December 2019) was searched to identify articles describing patients aged < 55-years harboring CAA or iCJD with previous history of neurosurgical procedures. Combinations of the following keywords were used: cerebral amyloid angiopathy; youth; dural graft; neurosurgical procedures; childhood; prion; iatrogenic Creutzfeldt-Jakob disease; amyloid-beta. 23 potentially eligible articles were considered.

\section{Results}

Thirty-seven case reports were retained for this review, yielding a total sample of twenty-nine patients (Table 1). Eight patients were excluded because they did not meet the age criteria (age $<55$ ). All of the selected patients underwent neurosurgical procedures decades prior to developing CAA. The median age of onset of symptoms was 36-years-old. The underlying pathology that prompted neurosurgical intervention was described in 18 patients and classified as traumatic brain injury (TBI) in 13 patients, brain tumors in 3,1 craniofacial malformation and 1 embolization. None of the patients had known predisposing mutations to develop early $A \beta$ pathology, such as mutations in APP, PSEN1 and PSEN2 genes. APOE genotype was $\varepsilon 3 / \varepsilon 3$ found in 6 patients, $\varepsilon 2 / \varepsilon 3$ in 3 and $\varepsilon 3 / \varepsilon 4$ in two of them.

\section{Discussion}

Human transmission of $A \beta$ pathology was initially postulated in 2015 [3], when an autopsy study of eight patients who died because of iCJD following treatment with cadaveric pituitary-derived growth hormone contaminated with prions, found moderate to severe grey matter and vascular $A \beta$ pathology in four of them. $A \beta$ deposition located in grey matter was similar to that usually observed in $A D$, and $A \beta$ deposition observed in the blood vessel walls was typical of CAA. None of those patients had genetic risk factors associated with early-onset $A D$. For the first time, it was suggested that iatrogenic transmission of $A \beta$ pathology might have happened in addition to ICJD, considering that the $A \beta$ deposition prevalence in controls of the same age is much lower than observed in that study. Later in 2016 Frontzek [8] investigated seven brains of patients who succumbed to iCJD after dural grafting related to previous neurosurgery and compared as controls 21 brains of age-matched patients with sporadic Creuzfelt-Jakob disease (sCJD). It was found that in five of seven iCJD brains, $A \beta$ was deposited in meningeal vessels as congofilic amyloid angiopathy. This was significantly more frequent than in the age-matched SCJD controls. Both studies' findings suggest that both human dural tissue grafts and pituitary extract are able to elicit $A \beta$ pathology decades later.
Unlike previous literature reviews [6] we included cases with coexistent CAA and iCJD after neurosurgical procedures and documented the use of dural graft when the information was available. There were 14 cases with CAA [1,2,5,9-11]4 with iCJD + CAA [8] and 11 with exclusively iCJD [8,12]. All patients with iCJD + CAA and those with exclusively iCJD developed their diseases after dural graft was implanted. However, dural graft was used in 4 out 14 patients who developed exclusively CCA. The latency period was longer in patients who developed CAA (mean $=32$-years) than in those who developed iCJD (mean = 14-years).

Despite the repeated description of cases of CAA following neurosurgical procedures the pathogenesis of $A \beta$ transmission remains unclear. Potential explanations include that both dural graft insertion and surgical instruments carrying traces of misfolded $A \beta$ protein [2], may be responsible for the development of CAA in patients with no genetic risk factors. On the other hand, the dysfunction of the lymphatic system or intramural peri-arterial drainage pathways, proposed as a possible explanation of sporadic CAA, could be triggered by neurosurgical procedures leading to the development of CAA [6]. However, all these theories remain untested in the early CAA pathogenesis.

\section{Conclusion}

Cerebral amyloid angiopathy (CAA) is an extremely rare pathology in young patients. There is no evidence in the literature of any patient $<55$-years old who developed CAA without known predisposing mutations and without personal history of neurosurgery. This fact suggests a possible transmission of amyloid- $\beta$ by a similar mechanism to acquired prion disease. This case report highlights the importance of considering CAA in the differential diagnosis of lobar intracerebral hemorrhage in young adults, especially with a past medical history of neurosurgery.

\section{References}

1. Hervé $D$, Porché $M$, Cabrejo $L$, et al. (2018) Fatal $A \beta$ cerebral amyloid angiopathy 4 decades after a dural graft at the age of 2 years. Acta Neuropathol (Berl) 135: 801-813.

2. Jaunmuktane Z, Quaegebeur A, Taipa R, et al. (2018) Evidence of amyloid- $\beta$ cerebral amyloid angiopathy transmission through neurosurgery. Acta Neuropathol (Berl) 135: 671-679.

3. Jaunmuktane Z, Mead S, Ellis M, et al. (2015) Evidence for human transmission of amyloid- $\beta$ pathology and cerebral amyloid angiopathy. Nature 525: 247-250.

4. Preusser M (2005) Alzheimer-type neuropathology in a 28-yearold patient with iatrogenic Creutzfeldt-Jakob disease after dural grafting. J Neurol Neurosurg Psychiatry 77: 413-416.

5. Banerjee G, Adams ME, Jaunmuktane Z, et al. (2019) Early onset cerebral amyloid angiopathy following childhood exposure to cadaveric dura. Ann Neurol 85: 284-290.

6. Hamaguchi T, Komatsu J, Sakai K, et al. (2019) Cerebral hemorrhagic stroke associated with cerebral amyloid angiopathy in young adults about 3 decades after neurosurgeries in their infancy. J Neurol Sci 399: 3-5. 
7. Nakayama Y, Mineharu Y, Arawaka Y, et al. (2017) Cerebral amyloid angiopathy in a young man with a history of traumatic brain injury: A case report and review of the literature. Acta Neurochir (Wien) 159: 15-18.

8. Frontzek K, Lutz MI, Aguzzi A, et al. (2016) Amyloid- $\beta$ pathology and cerebral amyloid angiopathy are frequent in iatrogenic CreutzfeldtJakob disease after dural grafting. Swiss Med Wkly 146: w14287.

9. Ehling R, Helbok R, Beer R, et al. (2012) Recurrent intracerebral haemorrhage after coitus: A case report of sporadic cerebral amyloid angiopathy in a younger patient. Eur J Neurol 19: e29-e31.
10. Giaccone G, Maderna E, Marucci G, et al. (2019) latrogenic early onset cerebral amyloid angiopathy 30 years after cerebral trauma with neurosurgery: Vascular amyloid deposits are made up of both $A \beta 40$ and $A \beta 42$. Acta Neuropathol Commun7: 70.

11. Purrucker JC, Hund E, Ringleb PA, et al. (2013) Cerebral amyloid angiopathy-an underdiagnosed entity in younger adults with lobar intracerebral hemorrhage? Amyloid 20: 45-47.

12. Cali I, Cohen ML, Haik S, et al. (2018) latrogenic CreutzfeldtJakob disease with Amyloid- $\beta$ pathology: An international study. Acta Neuropathol Commun 6: 5. 